\title{
Leaf extracts from Dendropanax morbifera Léveille mitigate mercury-induced reduction of spatial memory, as well as cell proliferation, and neuroblast differentiation in rat dentate gyrus
}

Woosuk Kim', Dae Young Yoo ${ }^{2}$, Hyo Young Jung ${ }^{1}$, Jong Whi Kim ${ }^{1}$, Kyu Ri Hahn' ${ }^{1}$, Hyun Jung Kwon ${ }^{3}$, Miyoung Yoo ${ }^{4}$, Sanghee Lee ${ }^{4}$, Sung Min Nam ${ }^{5}$, Yeo Sung Yoon ${ }^{1}$, Dae Won Kim ${ }^{3}$ and In Koo Hwang ${ }^{1 *}$ (D)

\begin{abstract}
Background: The brain is susceptible to methylmercury toxicity, which causes irreversible damage to neurons and glia and the leaf extract Dendropanax morbifera Léveille (DML) has various biological functions in the nervous system. In this study, we examined the effects of DML on mercury-induced proliferating cells and differentiated neuroblasts.

Methods: Dimethylmercury $(5 \mathrm{\mu g} / \mathrm{kg})$ and galantamine $(5 \mathrm{mg} / \mathrm{kg})$ was administered intraperitoneally and/or DML (100 $\mathrm{mg} / \mathrm{kg}$ ) was orally to 7-week-old rats every day for 36 days. One hour after the treatment, novel object recognition test was examined. In addition, spatial probe tests were conducted on the 6th day after 5 days of continuous training in the Morris swim maze. Thereafter, the rats were euthanized for immunohistochemical staining analysis with Ki67 and doublecortin and measurement for acetylcholinesterase (AChE) activity.
\end{abstract}

Results: Dimethylmercury-treated rats showed reduced discrimination index in novel object recognition test and took longer to find the platform than did control group. Compared with dimethylmercury treatment alone, supplementation with DML or galatamine significantly ameliorated the reduction of discrimination index and reduced the time spent to find the platform. In addition, the number of platform crossings was lower in the dimethylmercury-treated group than in controls, while the administration of DML or galantamine significantly increased the number of crossings than did dimethylmercury treatment alone. Proliferating cells and differentiated neuroblasts, assessed by Ki67 and doublecortin immunohistochemical staining was significantly decreased in the dimethylmercury treated group versus controls. Supplementation with DML or galantamine significantly increased the number of proliferating cells and differentiated neuroblasts in the dentate gyrus. In addition, treatment with dimethylmercury significantly increased AChE activity in hippocampal homogenates, while treatment with dimethylmercury+DML or dimethylmercury+galantamine significantly ameliorated this increase.

Conclusions: These results suggest that DML may be a functional food that improves dimethylmercuryinduced memory impairment and ameliorates dimethylmercury-induced reduction in proliferating cells and differentiated neuroblasts, and demonstrates corresponding activation of AChE activity in the dentate gyrus.

Keywords: Dendropanax morbifera extract, Mercury, Morris water maze, Neurogenesis, Hippocampus

\footnotetext{
*Correspondence: vetmed2@snu.ac.kr

${ }^{1}$ Department of Anatomy and Cell Biology, College of Veterinary Medicine,

and Research Institute for Veterinary Science, Seoul National University, Seoul

08826, South Korea

Full list of author information is available at the end of the article
}

(c) The Author(s). 2019 Open Access This article is distributed under the terms of the Creative Commons Attribution 4.0 International License (http://creativecommons.org/licenses/by/4.0/), which permits unrestricted use, distribution, and reproduction in any medium, provided you give appropriate credit to the original author(s) and the source, provide a link to the Creative Commons license, and indicate if changes were made. The Creative Commons Public Domain Dedication waiver (http://creativecommons.org/publicdomain/zero/1.0/) applies to the data made available in this article, unless otherwise stated. 


\section{Background}

Heavy metals such as mercury, lead, and cadmium are hazardous because they are bioaccumulated and biomagnified as they ascend the food chain. In particular, dimethylmercury $(\mathrm{MeHg})$, the most common cause of intoxication in humans [1], accumulates with the consumption of fish, including long-lived predatory species such as sharks and tuna. Absorbed $\mathrm{MeHg}$ enters the bloodstream, easily crosses the blood-brain barrier [2, 3], and is distributed to the brain, including the hippocampus and cerebellum [4]. Accumulated evidences demonstrated that Sprague-Dawley rats were most widely used animal models for $\mathrm{MeHg}$ toxicity in the brain. In addition, impairments of neurite outgrowth and cell migration was pronounced in the cells derived from male fetuses compared to cells from females [5]. Developmental $\mathrm{MeHg}$ exposure has been shown to impair memory during puberty [6, 7]; cognitive functions are impaired and ultrastructural abnormalities are observed in the dentate gyrus of adult rats exposed to $\mathrm{MeHg}$ [8]. In addition, treatment with $\mathrm{MeHg}$ impairs the cellular excitability and synaptic transmission by blocking the blocks calcium and sodium channels in rat hippocampal slices, while it does not affect the synaptic plasticity [9]. $\mathrm{MeHg}$ was found to reduce cell proliferation in the hippocampus at postnatal day 7 (P7), and significantly decrease granule cell layer population at P21 [6].

The hippocampus is a critical brain region involved in memory formation. Most of the granule cells in the hippocampal dentate gyrus are generated prenatally and intensive proliferation occurs during the first postnatal week, with differentiation into neurons occurring up to the third postnatal week $[10,11]$. In the adult brain, the active cells located in the subgranular zone of the dentate gyrus are able to proliferate, migrate to granule cell layer, and differentiate into neuroblasts. Neuroblasts are integrated into granule cells and contribute to learning and memory processes [12]. Hence, the facilitation of cell proliferation and neuroblast differentiation can be targets to promote hippocampal function and regenerative processes in neurological disorders such as Alzheimer's disease.

Medicinal plants have been widely investigated because of their ability to cure various disease states and maintain health with little to no adverse effects [13, 14]. Dendropanax morbifera is an endemic and evergreen plant in the Southern Korea and its leaves, stems, and roots are widely used in folk medicine for the treatment of skin problems and headache [15]. Dendropanax morbifera was also shown to ameliorate neuronal damage in an animal model of Parkinson's disease by reducing neuroinflammation [16] and memory deficits in a chemically-induced aging model by decreasing pro-inflammatory cytokine levels in the hippocampus [17]. In addition, Dendropanax morbifera also alleviated hippocampal impairment in cadmium- and mercury-induced neurotoxicity rats $[18,19]$ and reversed the calcium-induced reduction of proliferating cells and differentiated neuroblasts in the hippocampus [20]. Previously, we demonstrated that $\mathrm{MeHg}$ increased oxidative stress in the hippocampus, with the administration of an extract of Dendropanax morbifera leaves (DML) significantly ameliorating this increase [19]. Treatment with DML shows fewer side effects such as cutaneous atrophy and erythema compared to that in the repeated dexamethasone use [21]. In addition, randomized, doubleblinded trial conducted by our colleagues demonstrates there are minimal adverse effects in humans [22]. However, no studies have been reported on the preventive potentials of Dendropanax morbifera on MeHg-induced memory deficits, decreases in proliferating cells and differentiated neuroblasts in the hippocampus. The present study, therefore, investigated the effects of DML and $\mathrm{MeHg}$ on hippocampal memory, proliferating cells and differentiated neuroblasts in the dentate gyrus of the hippocampus.

\section{Methods \\ Experimental animals}

We purchased the male Sprague-Dawley rats (6 weeks of age) from Orient Bio Inc. (Seongnam, South Korea). Two animals were housed per cage at constant temperature $\left(22 \pm 2{ }^{\circ} \mathrm{C}\right)$ and humidity $(60 \pm 5 \%)$ on a 12 / $12 \mathrm{~h}$ light/dark cycle with ad libitum access to food and water. The experimental protocols and ethics were approved the Institutional Animal Care and Use Committee of Seoul National University (SNU-130911-4 and SNU-151104-1). All experiments were conducted with an effort to minimize the number of animals used and the physiological stress caused by the procedures employed. All experimental procedures were conducted according to ARRIVE guidelines [23].

\section{Preparation of DML}

DML were obtained by ethanol extraction as previously described [19]. Briefly, DML was extracted with $80 \%$ ethanol, and then refluxed three times for $2 \mathrm{~h}$. Centrifugation was conducted at $10,000 \times g$ for $30 \mathrm{~min}$ to remove the insoluble materials and a powder was obtained after concentration and freeze-drying.

\section{Quantification of phenolic compounds in DML by HPLC} HPLC analysis of DML was performed described by Hyun et al. [24]. DML sample was dissolved in $80 \%$ methanol solution and filtered with $0.2-\mu \mathrm{m}$ syringe filter (TITAN, nylon) for high-performance liquid chromatography (HPLC). 
The Thermo ACELLA HPLC (Thermoscientific, Waltham, MA, USA) system was used in this study with C18, $2.1 \times 100 \mathrm{~mm}, 2.6-\mu \mathrm{m}$ column (Thermo Accucore). Flow rate of solvent was set with $500 \mu \mathrm{L} / \mathrm{min}$ and every $4 \mu \mathrm{L}$ of the sample was injected with 25 min of runtime. Rutin, chlorogenic acid, (+)-catechin, ferulic acid, quercetin, and myricetin were dissolved in methanol and the concentration of these compounds was measured by calculation of peak areas of samples with the calibration curve of the standards.

\section{Administration of $\mathrm{MeHg}$ and DML}

Animals were randomly divided into four groups $(n=15 /$ group): 1) distilled water- and saline-treated control group (control group), 2) distilled water- and $\mathrm{MeHg}$-treated group, 3) $\mathrm{MeHg}$ and DML (MeHg+DML)-treated group, and 4) $\mathrm{MeHg}$ and galantamine (MeHg+GAL)-treated group. Distilled water, $100 \mathrm{mg} / \mathrm{kg} \mathrm{DML}$, and $5 \mathrm{mg} / \mathrm{kg} \mathrm{GAL}$ was orally administered to 7-week-old rats once daily for 36 days, while saline and $5 \mu \mathrm{g} / \mathrm{kg} \mathrm{MeHg}$ was treated intraperitoneally. GAL inhibits acetylcholinesterase activity and shows ameliorative effects on the reduction of hippocampal neurogenesis after cadmium exposure [20] and scopolamine toxicity $[25,26]$.

\section{Novel object recognition test}

The test was performed as described in our previous study with minor modifications [20]. Briefly, the testing apparatus was made with black acryl with $80 \times$ $60 \times 40 \mathrm{~cm}$ open box. The objects were purchased with metallic to avoid the displacements by the rats due to their weight.

On 34th day of treatment with DML, GAL, or MeHg, $1 \mathrm{~h}$ after treatment, rats from each group $(n=5$ per group) were freed to explore the apparatus for $2 \mathrm{~min}$ for adaption. The next day, 2-min training and testing trials were conducted $1 \mathrm{~h}$ following treatment with DML, GAL, or MeHg. During the training trial, animals were allowed to explore two identical objects placed in opposite corners of the apparatus. After $1 \mathrm{~h}$ interval, one familiar object was replaced into new (novel) object and rats were exposed to the objects during testing trial. Exploration time was measured when the nose of rat is located in no more than $2 \mathrm{~cm}$ of objects. Total spent time was calculated in both trials to explore the familiar and new objects. To elucidate the rats' discrimination of two objects, discrimination index was also calculated by ratio of exploration time in each object vs. total time spent exploring the two objects in testing trial.

\section{Morris water maze test}

On 29th day of treatment with DML, GAL, or $\mathrm{MeHg}$, the Morris water maze test was conducted with Charles V Voorhees \& Michael T Williams method. Animals were placed in the water maze pool, which is divided into four quadrants (A, B, C, and D). Each animal ( $n=$ 10 per group) was randomly trained once in each quadrant, for a total of four times a day, for 5 consecutive days. Escape latency was automatically checked as the time elapsed to find the platform and escape from the water.

On 35th day of treatment with DML, GAL, or MeHg, $1 \mathrm{~h}$ after treatment, the platform was removed and the rats entered the water from a different quadrant. In this spatial probe test, which measures learning and memory ability, rats were observed and recorded for $30 \mathrm{~s}$ to measure the time spent and the number of crossings in the quadrant at the original platform position.

\section{Tissue processing}

One day after Morris water maze test, $1 \mathrm{~h}$ after treatment with DML, GAL, or $\mathrm{MeHg}$, rats $(n=10$ in each group) were euthanized with overdose of $2 \mathrm{~g} / \mathrm{kg}$ of urethane (Sigma-Aldrich, St. Louis, MO, USA). Transcardiac perfusion was performed with $0.1 \mathrm{M}$ phosphatebuffered saline (PBS, pH 7.4), and followed by $4 \%$ paraformaldehyde in $0.1 \mathrm{M}$ PBS ( $\mathrm{pH} 7.4$ ), as previously described [20,27]. Serial coronal hippocampal sections $(30 \mu \mathrm{m})$ were made using a cryostat (Leica, Wetzlar, Germany) located 3.00 and $4.08 \mathrm{~mm}$ posterior to bregma in the rat brain atlas [28].

\section{Immunohistochemistry}

Tissue sections, located $90 \mu \mathrm{m}$ apart, were used for immunohistochemical staining. Briefly, primary antibodies were used as follows; rabbit anti-Ki67 antibody (1:1000; Abcam, Cambridge, UK) and rabbit anti-doublecortin (DCX) antibody (1:5000; Abcam). The antigen-antibodyhorseradish peroxidase complex is allowed to react with a 3,3'-diaminobenzidine tetrachloride (Sigma) substrate for staining.

\section{Data analysis}

DCX immunoreactivity in hippocampal dentate gyrus was analyzed ImageJ software v. 1.50 (National Institutes of Health, Bethesda, MD, USA), as described previously [20]. Briefly, the intensity of DCX immunoreactivity was measured according to pixel resolution with 256 Gy levels and relative optical density (ROD) was calculated by the following formula: $\mathrm{ROD}=\log _{10}(256 /$ mean gray level).

The number of Ki67- and DCX-immunoreactive cells were counted in dentate gyrus using an analysis system (OPTIMAS software version 6.5; CyberMetrics ${ }^{\circ}$ Corporation, Phoenix, AZ, USA; magnification, 100×) as described previously [20]. The image was converted to a gray-scale image and Ki67-positive nuclei and DCXimmunoreactive neuroblasts were automatically 
selected according to the intensity of the immunohistochemical staining.

\section{Measurement of AChE activity in the hippocampus} One day after novel object recognition test, $1 \mathrm{~h}$ after treatment with DML, GAL, or MeHg, rats $(n=5$ in each group) were euthanized to measure $\mathrm{AChE}$ activity in synaptosomes as described previously [20]. Briefly, hippocampi in each animal were quickly removed from the brain and a quick-freeze was conducted in liquid nitrogen for $15 \mathrm{~min}$. Synaptosomes in hippocampal tissues were freshly isolated with discontinuous Percoll gradient [29]. The AChE enzyme activity was measured with the formation of the yellow anion, 5,5' -dithio-bis-acid nitrobenzoic [30].

\section{Statistical analysis}

The data were expressed as mean with standard error of the mean or standard deviation. Mean differences among the groups were analyzed with Student $t$-test or one-way analyses of variance followed by Bonferroni's post-hoc test using GraphPad Prism 5.01 software (La Jolla, CA, USA). Statistical significance was set at $p<0.05$.

\section{Results}

\section{Quantification of phenolic compounds in DML}

For standardization and to observe the functional components of DML, most abundant 6 phenolic compounds in DML described by Hyun et al. [24] were measured. Rutin was most abundantly observed in the DML, while myricetin was lowest content in the DML. Chlorogenic acid, (+)-catechin, and ferulic acid was observed with $7.2-18.3 \mu \mathrm{g} / \mathrm{g}$ in DML (Table 1).

\section{Effects of MeHg and DML on Morris water maze test}

In all groups, escape latency progressively decreased from days 1 to 5 , although the swimming speed did not show any significant differences among groups. There was a significantly higher escape latency in the MeHg-treated group compared the control group on

Table 1 Contents of phenolic compounds in DML are analyzed by HPLC method. Among these 6 phenolic compounds, rutin is the highest content and myricetin is lowest in DML. Values are mean \pm standard deviation $(n=3)$

\begin{tabular}{lll}
\hline No. & Phenolic compound & Concentration $(\mu \mathrm{g} / \mathrm{g})$ \\
\hline 1 & Rutin & $26.8 \pm 0.4$ \\
2 & Chlorogenic acid & $18.3 \pm 0.6$ \\
3 & $(+)$-Catechin & $14.9 \pm 0.1$ \\
4 & Ferulic acid & $12.8 \pm 0.4$ \\
5 & Quercetin & $7.2 \pm 0.3$ \\
6 & Myricetin & $4.9 \pm 0.2$ \\
\hline
\end{tabular}

days 4 and 5 . MeHg+DML-treated rats showed a significant reduction in escape latency on the days 4 and 5 compared to the MeHg-treated group. In addition, $\mathrm{MeHg}+\mathrm{GAL}$-treated rats had similar escape latency in all experimental periods with that in the $\mathrm{MeHg}$ + DML-treated rats (Fig. 1a and b).

On day 6 , the number of crossings at the original platform location and the target quadrant time were measured. $\mathrm{MeHg}$-treated rats showed a significant reduction in the number of crossings and target quadrant time compared to the control group. However, MeHg+DML and $\mathrm{MeHg}+\mathrm{GAL}$ treatment significantly reversed these effects (Fig. 1c and d).

\section{Effects of MeHg and DML on novel object recognition test}

In training trial, all animals did not show any significant differences on exploration time in two same familiar objects (Fig. 2a). In the testing trial, rats in control group showed significant increases in exploration time in new object than in familiar object. MeHg-treated rats showed a reduction in exploration time in new object compared to that in the control group, and there were no significant differences on exploration time spent between familiar and new object. Rats in MeHg+DML- and $\mathrm{MeHg}+\mathrm{GAL}$-treated groups spent much more time in new object than in the familiar object compared to that in the MeHg-treated group. However, statistical significance was not detected between groups (Fig. 2b). Discrimination index showed significant differences between groups. In the $\mathrm{MeHg}$-treated group, discrimination index was significantly decreased compared to that in the control group. In the MeHg+DML-treated group, discrimination index was significantly increased compared to that in the $\mathrm{MeHg}$ group, although discrimination index in this group was significantly lower than in the control group. In the $\mathrm{MeHg}+\mathrm{GAL}$-treated group, discrimination index was similarly observed compared to that in the MeHg+DML-treated group (Fig. 2c).

\section{Effects of MeHg and DML on cell proliferation in the dentate gyrus}

In all groups, Ki67-positive nuclei were mainly observed in the subgranular zone of dentate gyrus (Fig. 3a-d). However, there were significant differences in the number of Ki67-positive nuclei in the dentate gyrus among groups (Fig. 3e). In the MeHg-treated group, the number of Ki67-positive nuclei was significantly decreased to $57.0 \%$ of that observed in the control group. However, in the MeHg+DML- and MeHg+GAL-treated groups, the numbers of Ki67-positive nuclei were significantly increased to 145.8 and $138.2 \%$ of that observed in the $\mathrm{MeHg}$-treated group, respectively, although the number 


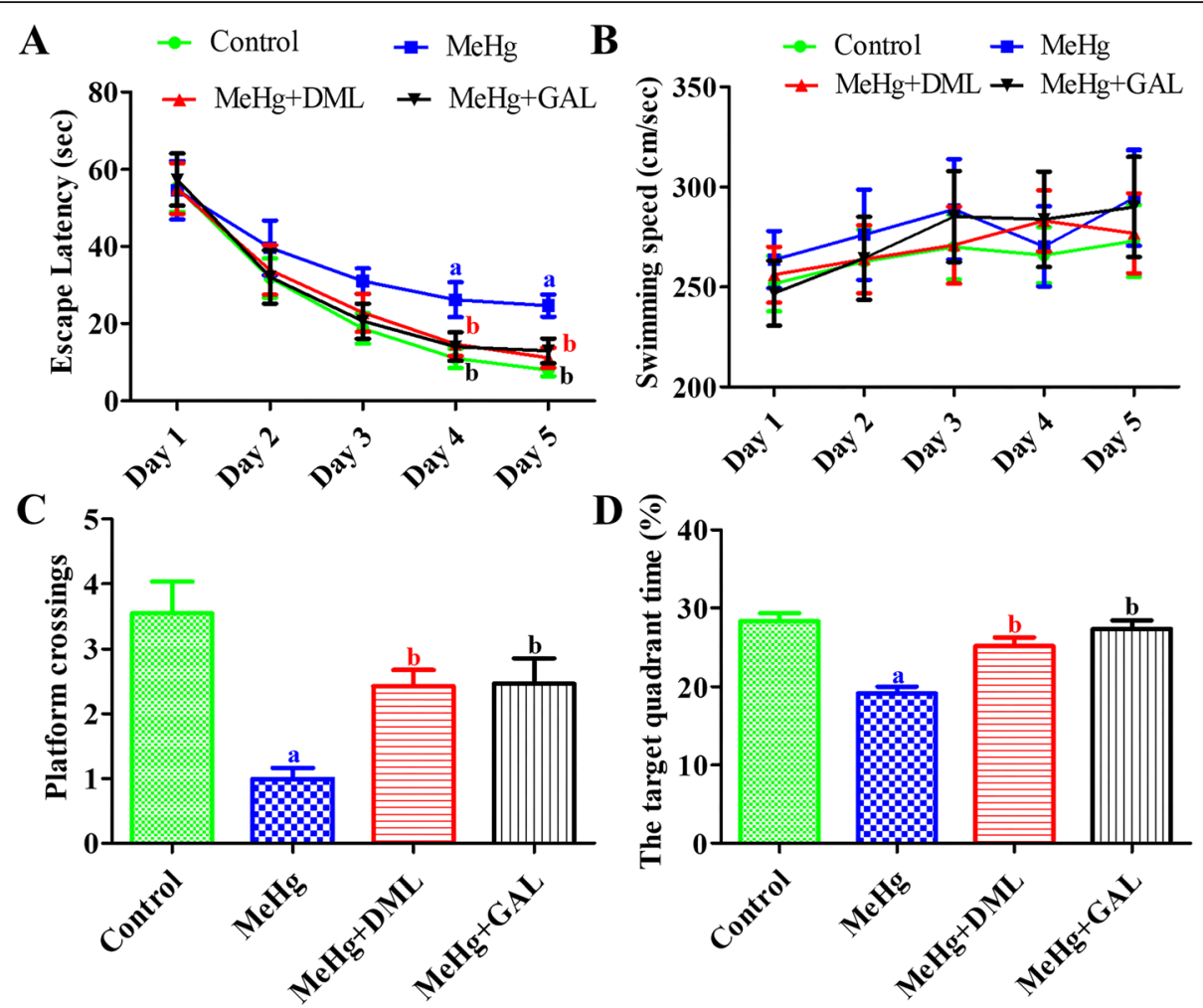

Fig. 1 Escape latency training trials $(\mathbf{a})$, average speed $(\mathrm{cm} / \mathrm{sec})(\mathbf{b})$, frequency of target crossing $(\mathbf{c})$, and time spent in the correct quadrant (d) of the control group, dimethylmercury (MeHg)-treated group, Dendropanax morbifera leaf extract treated group with $\mathrm{MeHg}(\mathrm{MeHg}+\mathrm{DML})$, and galantamine (GAL) treated group with MeHg (MeHg + GAL) in the Morris water maze task $\left(n=10 ;{ }^{a} p<0.05\right.$, vs. control group; ${ }^{b} p<$ MeHg-treated group). Values are mean \pm standard error of the mean. Note that escape latency is highest in the MeHg group on days 4 and 5 and it is significantly decreased in the MeHg+DML and MeHg+GAL group on the days 4 and 5 . In addition, on the day 6, number of crossings and target quadrant time is significantly decreased in the $\mathrm{MeHg}$ group compared to that in the control group and they significantly ameliorated in MeHg $+\mathrm{DML}$ and MeHg+GAL group

was significantly lower in the MeHg+GAL-treated group than in control group (Fig. 3).

\section{Effects of MeHg and DML on neuroblast differentiation in the dentate gyrus}

In the control group, DCX immunoreactive cell bodies were found in the subgranular zone of the dentate gyrus, and their dendrites crossed the granule cell layer and extended into the molecular layer (Fig. 4a and b). The mean number of DCX immunoreactive neuroblasts in controls was $115.6 \pm 14.9$ per section (Fig. 4i). In the MeHg-treated group, fewer DCX immunoreactive neuroblasts and less DCX immunoreactive fibers were detected in the dentate gyrus than in the control group (Fig. 4c and d). In the MeHg-treated group, the number of DCX immunoreactive neuroblasts and DCX immunoreactivity were significantly decreased in the dentate gyrus by 52.3 and $46.7 \%$ than that of the control group, respectively (Fig. 4i and j). In the $\mathrm{MeHg}+\mathrm{DML}-$ and $\mathrm{MeHg}+\mathrm{GAL}$-treated groups, more DCX immunoreactive neuroblasts were observed, with well-developed DCX immunoreactive dendrites than the MeHg-treated group
(Fig. 4e, f, g, and h). In the MeHg+DML-treated group, the number of DCX immunoreactive neuroblasts and DCX immunoreactivity were significantly increased by 152.8 and $170.5 \%$ than that in the $\mathrm{MeHg}$-treated group, respectively (Fig. 4i and $j$ ). In the $\mathrm{MeHg}+\mathrm{GAL}$-treated group, similar number and immunoreactivity of DCX positive neuroblasts were observed in the dentate gyrus compared to that in the $\mathrm{MeHg}+\mathrm{DML}$-treated group (Fig. $4 \mathrm{i}$ and j).

\section{Effects of MeHg and DML on AChE activity in hippocampal homogenates}

In the control group, AChE activity was $4.50 \mu \mathrm{mol}$ $\mathrm{AcSCh} / \mathrm{h} / \mathrm{mg}$ protein in hippocampal homogenates. In the $\mathrm{MeHg}$-treated group, AChE activity was significantly increased by $147.4 \%$ of control group, while in the MeHg+DML- and MeHg+GAL-treated groups, AChE activity was significantly decreased by 71.2 and $68.5 \%$ of the MeHg-treated group, respectively, and was similar to the levels observed in the control group (Fig. 5). 


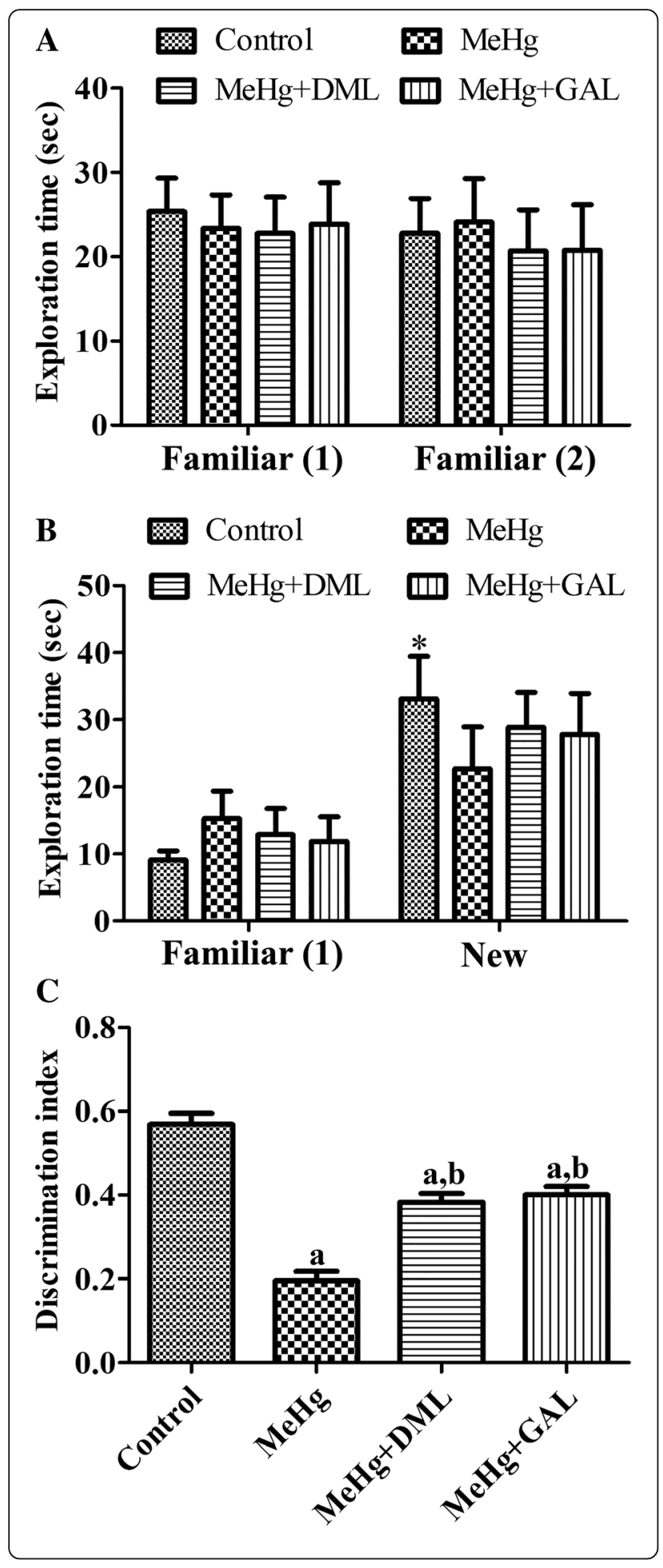

Fig. 2 Exploration time in training (A) and testing trials (B), and discrimination index (C) in the control group, dimethylmercury (MeHg)-treated group, Dendropanax morbifera leaf extract treated group with $\mathrm{MeHg}(\mathrm{MeHg}+\mathrm{DML})$, and galantamine $(\mathrm{GAL})$ treated group with $\mathrm{MeHg}(\mathrm{MeHg}+\mathrm{GAL})\left(n=5\right.$ per group; ${ }^{*} p<0.05$, vs. familiar object; ${ }^{a} p<0.05$, vs. control group; ${ }^{b} p<$ MeHg-treated group). All data are shown as $\%$ exploration time \pm standard error of the mean. Note that training trial does not show any remarkable differences on exploration time. In contrast, control group shows increased exploration time in new object compared to that in the MeHg group, while exploration time is decreased in MeHg group. In the $\mathrm{MeHg}+\mathrm{DML}$ and $\mathrm{MeHg}+\mathrm{GAL}$ groups, exploration time in new object is increased. In addition, discrimination index is significantly decreased in the MeHg group compared to that in the control group. In the MeHg+DML and MeHg+GAL group, discrimination index is remarkably increased compared to that in the MeHg group

\section{Discussion}

MeHg shows toxic effects on human health, and prenatal exposure to mercury reduces the number of neuron and affects cytoarchitecture [31, 32]. Previous studies have shown that exposure to low or high levels of $\mathrm{MeHg}$ through the diet may accumulate in the hippocampus $[4,20,33]$ because $\mathrm{MeHg}$ can easily cross the blood-brain barrier and then readily converted to an inorganic form in the brain $[2,3]$. Studies have also demonstrated that early postnatal exposure to $\mathrm{MeHg}$ induces deficits in spatial memory $[6,7,34]$. In the present study, we observed that the administration of MeHg to adolescent to young-adult rats showed deficits in spatial memory in the Morris water maze test and novel object recognition test. In addition, supplementation with DML ameliorated the MeHg-induced impairments in the rats. We and our colleagues have previously demonstrated that DML or the root extract from Dendropanax morbifera ameliorated cadmium- [20] or D-galactose-induced [17] memory deficits in experimental animals, while an ethyl acetate fraction from Dendropanax morbifera mitigated high fat diet-induced cognitive impairments [35].

In the present study, we examined proliferating cells and differentiated neuroblasts in the dentate gyrus because stimulation and impairments of hippocampal neurogenesis are closely related to hippocampusdependent learning and memory [36-38]. During the postnatal development of the hippocampus, exposure to $\mathrm{MeHg}(0.6 \mu \mathrm{g} / \mathrm{g}$, subcutaneously) reduced hippocampal neurogenesis [39]. Similarly, $\mathrm{MeHg}$ exposure at P7 led to a marked decrease in the number of bromodeoxyuridine-positive cells by $29 \%$ in the granule cell layer and by $22 \%$ in the hilar region at P21 [6]. In the present study, treatment of rats with $5 \mu \mathrm{g} / \mathrm{g}$ $\mathrm{MeHg}$ significantly decreased proliferating cells and differentiated neuroblasts in the dentate gyrus, assessed by Ki67 and DCX immunohistochemistry, in adolescent/young-adult rats. In addition, the supplementation with DML significantly ameliorated the 

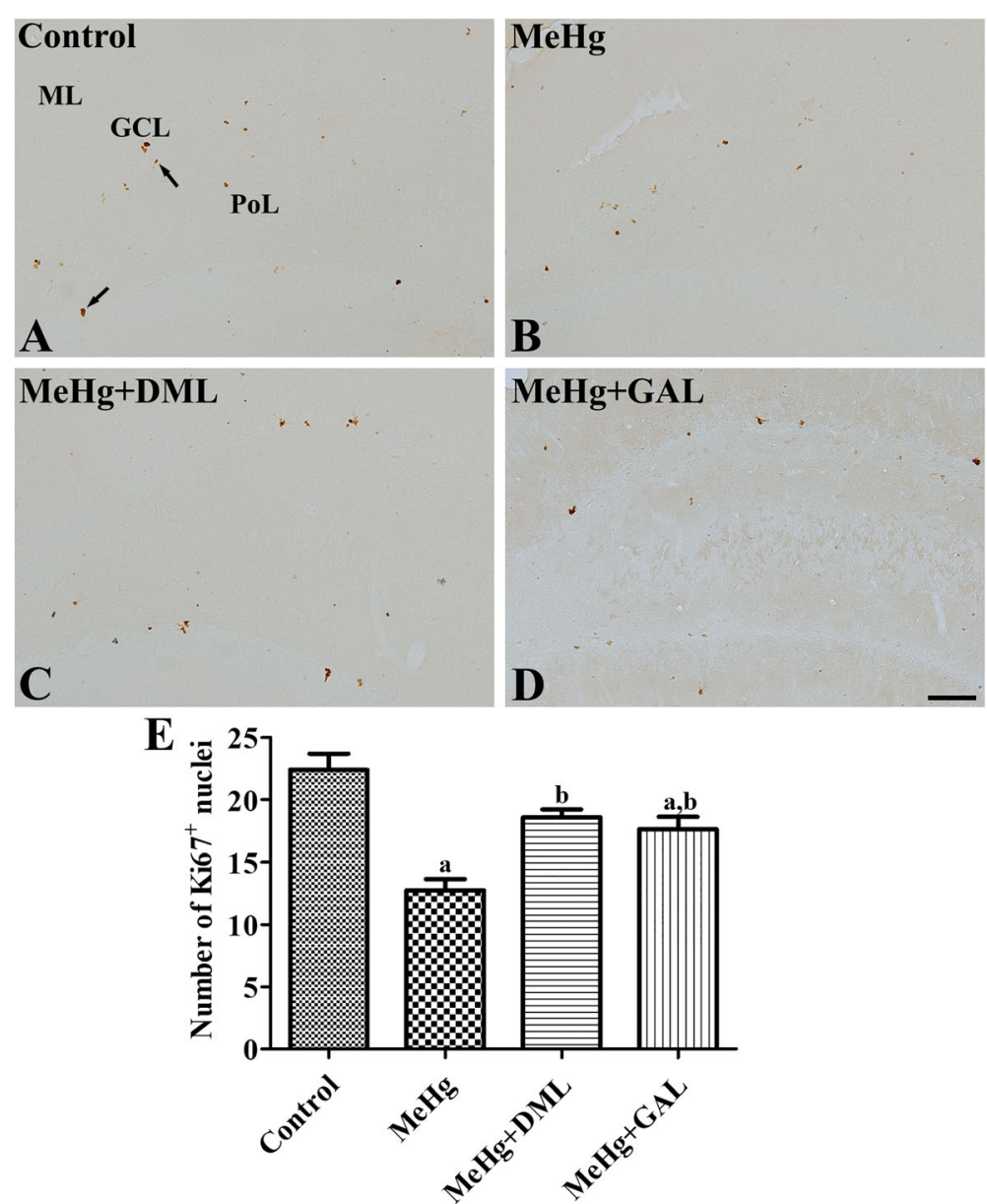

Fig. 3 Immunohistochemistry for Ki67 in the dentate gyrus of the control group (a), dimethylmercury (MeHg)-treated group (b), Dendropanax morbifera leaf extract treated group with $\mathrm{MeHg}(\mathrm{MeHg}+\mathrm{DML})(\mathbf{c})$, and galantamine (GAL) treated group with $\mathrm{MeHg}(\mathrm{MeHg}+\mathrm{GAL})(\mathbf{d})$. In the control group, Ki67 positive nuclei (arrows) are mainly observed in the subgranular zone of the dentate gyrus. Note that few Ki67 positive nuclei are found in the MeHg-treated group, while in the MeHg + DML-treated group, Ki67 positive nuclei are relatively abundant. GCL, granule cell layer; ML, molecular layer; PoL, polymorphic layer. Scale bar $=100 \mu \mathrm{m}$. (e): Quantitative analysis of Ki67 positive nuclei number per section in the control, MeHg-treated, and MeHg+DML-treated rats using an image analyzer ( $n=5$ per group; ap <0.05, vs. control group; bp < MeHg-treated group). Values are mean \pm standard error of the mean

MeHg-induced reduction of proliferating cells and differentiated neuroblasts in the dentate gyrus. In a previous study, we demonstrated significant increases in these cells in the dentate gyrus after treatment with the root extract from Dendropanax morbifera in normal (naïve) mice; furthermore, supplementation with Dendropanax morbifera ameliorated the cadmium-induced reduction in proliferating cells and differentiated neuroblasts [20].

AChE catalyzes the breakdown of acetylcholine and inhibition of AChE is a major target for Alzheimer's disease. In the present study, we also measured AChE activity in hippocampal synaptosomes. Exposure to $\mathrm{MeHg}$ produced significantly higher levels of AChE activity compared to that in the control group. This result is consistent with a previous study showing that subchronic treatment with mercury chloride increased AChE activity in the hippocampus by $125 \%$ than that in the control group [40]. Supplementation with DML significantly decreased $\mathrm{MeHg}$-induced activation of AChE activity in hippocampal synaptosomes. This result is consistent with that of a previous study demonstrating that administration of an ethyl acetate fraction from Dendropanax morbifera lowered AChE activity compared with that in the high-fat diet fed group [35]. Furthermore, inhibition of $\mathrm{AChE}$ by donepezil or GAL increased proliferating cells or hippocampal neurogenesis in the dentate gyrus of vascular dementia rats or normal mice [41-43]. In the present study, we confirmed that GAL also reduced MeHg-induced memory impairments and reduction of proliferating cells and differentiated neuroblasts in the dentate gyrus and DML 

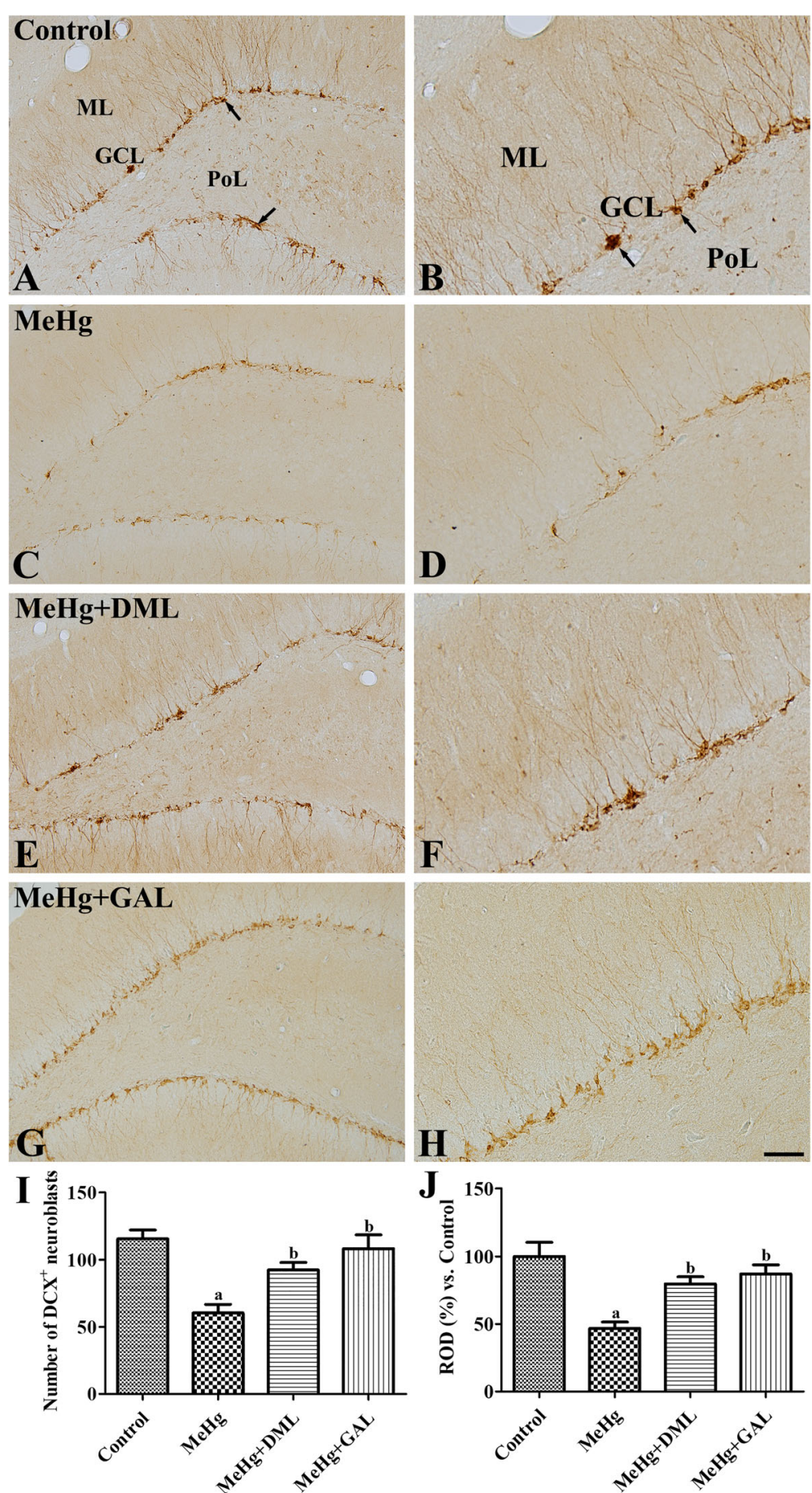

Fig. 4 Immunohistochemistry for doublecortin (DCX) in the dentate gyrus of the control group (a and $\mathbf{b}$ ), dimethylmercury (MeHg)-treated group (c and $\mathbf{d}$ ), Dendropanax morbifera leaf extract treated group with MeHg (MeHg+DML) (e and $\mathbf{f}$ ), and galantamine (GAL) treated group with MeHg (MeHg+GAL) ( $\mathbf{g}$ and $\mathbf{h}$ ). In the control group, DCX immunoreactive neuroblasts are abundantly in the dentate gyrus. Note that DCX immunoreactive neuroblasts are few and their dendrites are poorly developed in the MeHg-treated group. In the MeHg+DML-treated group, DCX immunoreactive neuroblasts are abundant and have well-developed dendrites. GCL, granule cell layer; ML, molecular layer; PoL, polymorphic layer. Scale bar $=100 \mu \mathrm{m}(\mathbf{a}, \mathbf{c}, \mathbf{e}$, and $\mathbf{g}), 50 \mu \mathrm{m}(\mathbf{b}, \mathbf{d}, \mathbf{f}$, and $\mathbf{h})$. (i and $\mathbf{j})$ : Quantitative analysis of DCX immunoreactive neuroblasts. The number per section and the relative optical densities (RODs), expressed as a percentage of the value representing the DCX immunoreactivity in the dentate gyrus of the control group, are also shown ( $n=5$ per group; $a p<0.05$, vs. control group; $b p<$ MeHgtreated group). Values are mean \pm standard error of the mean 


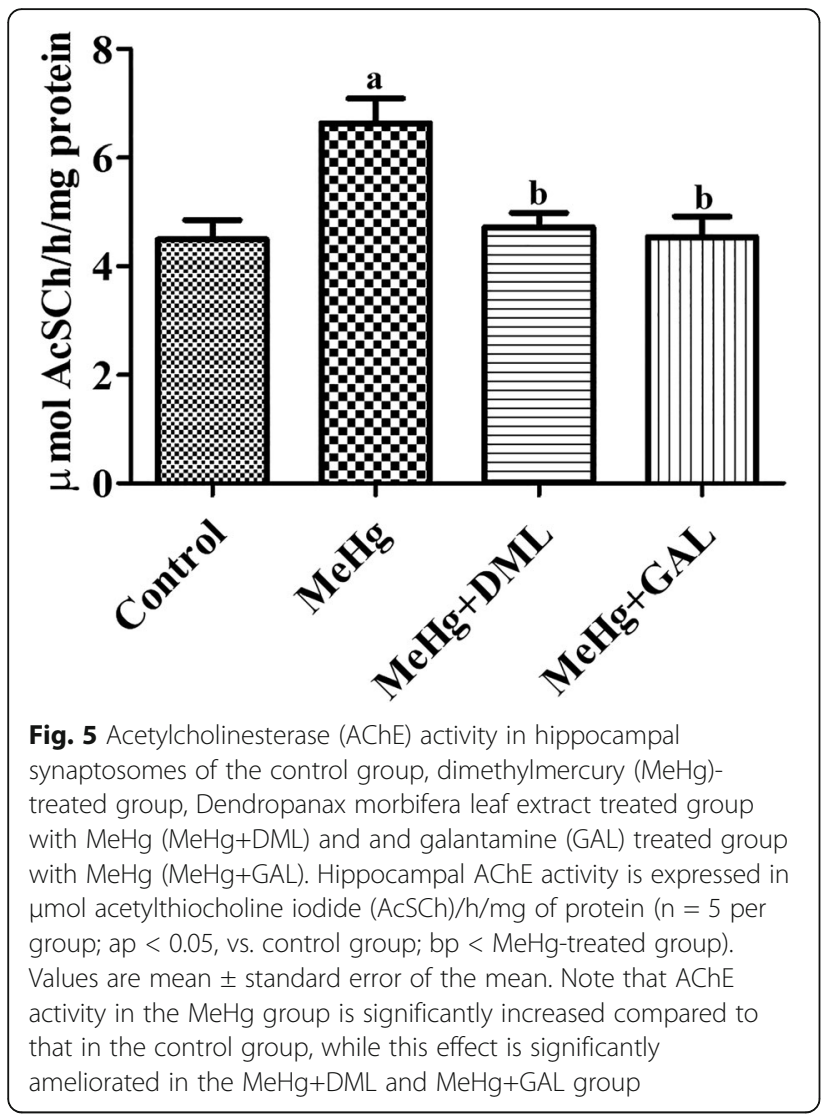

has comparable effects on hippocampal functions compared to that in the GAL.

In the present study, we also analyzed the contents of phenolic compounds by HPLC system demonstrated by Hyun et al. [24]. They observed analyzed 22 phenolic compounds in DML from green and senescent leaves [24] and we selected most abundant polyphenols in the present study and observed rutin and chlorogenic acid are most abundant in the DML. This result was supported by previous studies that rutin could decrease $\mathrm{MeHg}$ bioaccessibility in vitro digestion experiment [44]. Chlorogenic acid protects hippocampal neurons from aluminum-induced cytotoxicity by antioxidant actions [45]. In addition, chlorogenic acid ameliorates the scopolamine-induced amnesia in mice by anti-AChE and antioxidant activities [46]. In the present study, we used $80 \%$ ethanol solution for extraction of DML and Choi et al. observed highest levels of rutin and chlorogenic acid at $80 \%$ ethanol extracts [47]. In addition, they observed highest scavenging and reducing power activities at $80 \%$ ethanol extracts of DML [47]. In other components, myricetin, although the lowest contents in six polyphenols in DML, has antioxidant effects of MeHg-induced oxidative stress such as formation of reactive oxygen species and lipid peroxidation [48].

\section{Conclusions}

DML ameliorated MeHg-induced memory deficits, an effect that was associated with DML-induced cell proliferation and neuroblast differentiation, as well as lowered AChE activity, in the hippocampus.

\section{Abbreviations \\ AChE: Acetylcholinesterase; AcSCh: Acetylthiocholine iodide; \\ DCX: Doublecortin; DML: Extract of Dendropanax morbifera leaves; \\ GAL: Galantamine; HPCL: High-performance liquid chromatography; \\ IP: Intraperitoneal; MeHg: Dimethylmercury; P7: Postnatal day 7; \\ PBS: Phosphate-buffered saline; ROD: Relative optical density}

\section{Acknowledgements \\ Not applicable.}

\section{Funding}

This work was supported by the Basic Science Research Program through the National Research Foundation of Korea (NRF) funded by the Ministry of Education (NRF-2016R1A2B4009156). In addition, this work was supported by the Promising-Pioneering Researcher Program through Seoul National University (SNU) in 2015 and by the Research Institute for Veterinary Science, Seoul National University. The founding sponsors had no role in the study design, performance, data collection and analysis, decision to publish, or preparation/writing of the manuscript.

\section{Availability of data and materials}

The datasets and supporting materials including leaf extracts from Dendropanax morbifera Léveille generated during and/or analyzed during the current study are available from the corresponding author on reasonable request.

\section{Authors' contributions}

WK, DYY, HYJ, JWK, KRH, HJK, MY, SL, SMN, YSY, DWK, and IKH conceived the study. WK and IKH designed the study and wrote the manuscript. WK, DYY, $\mathrm{HYJ}$, JWK, and KRH conducted behavioral and morphological experiments. HJK and DWK conducted the biochemical experiments. MY and SL analyzed the polyphenols. DYY, SMN, and YSY participated in designing and discussing the study. All authors have read and approved the final manuscript.

\section{Ethics approval and consent to participate}

Animal handling and care conformed to the guidelines of current international laws and policies (National Institutes of Health Guide for the Care and Use of Laboratory Animals, Publication No. 85-23, 1985, revised 1996) and were approved by the Institutional Animal Care and Use Committee of Seoul National University (SNU-130911-4 and SNU-151104-1).

Consent for publication

Not applicable.

\section{Competing interests}

The authors declare that they have no competing interests.

\section{Publisher's Note}

Springer Nature remains neutral with regard to jurisdictional claims in published maps and institutional affiliations.

\section{Author details}

${ }^{1}$ Department of Anatomy and Cell Biology, College of Veterinary Medicine, and Research Institute for Veterinary Science, Seoul National University, Seoul 08826, South Korea. ${ }^{2}$ Department of Anatomy, College of Medicine,

Soonchunhyang University, Cheonan, Chungcheongnam 31151, South Korea. ${ }^{3}$ Department of Biochemistry and Molecular Biology, Research Institute of Oral Sciences, College of Dentistry, Gangneung-Wonju National University, Gangneung 25457, South Korea. ${ }^{4}$ Korea Food Research Institute, Jeollabuk-do 55365, South Korea. ${ }^{5}$ Department of Anatomy, College of Veterinary Medicine, Konkuk University, Seoul 05030, South Korea. 


\section{Received: 13 September 2018 Accepted: 17 April 2019}

\section{Published online: 02 May 2019}

\section{References}

1. Risher JF, Amler SN. Mercury exposure: evaluation and intervention the inappropriate use of chelating agents in the diagnosis and treatment of putative mercury poisoning. Neurotoxicology. 2005;26:691-9.

2. Yoshida M, Shimada E, Arai F, Yamamura Y. The relation between mercury levels in brain and blood or cerebrospinal fluid (CSF) after mercury exposure. J Toxicol Sci. 1980;5:243-50.

3. Harada M. Minamata disease: methylmercury poisoning in Japan caused by environmental pollution. Crit Rev Toxicol. 1995;25:1-24.

4. Fujimura M, Usuki F. Site-specific neural hyperactivity via the activation of MAPK and PKA/CREB pathways triggers neuronal degeneration in methylmercury-intoxicated mice. Toxicol Lett. 2017;271:66-73.

5. Edoff K, Raciti M, Moors M, Sundström E, Ceccatelli S. Gestational age and sex influence the susceptibility of human neural progenitor cells to low levels of MeHg. Neurotox Res. 2017;32:683-93.

6. Falluel-Morel A, Sokolowski K, Sisti HM, Zhou X, Shors TJ, Dicicco-Bloom E. Developmental mercury exposure elicits acute hippocampal cell death, reductions in neurogenesis, and severe learning deficits during puberty. J Neurochem. 2007;103:1968-81.

7. Karpova NN, Lindholm JS, Kulesskaya N, Onishchenko N, Vahter M, Popova D, Ceccatelli S, Castrén E. TrkB overexpression in mice buffers against memory deficits and depression-like behavior but not all anxiety- and stress-related symptoms induced by developmental exposure to methylmercury. Front Behav Neurosci. 2014;8:315.

8. Lu Z, Wu J, Cheng G, Tian J, Lu Z, Bi Y. Methylmercury chloride damage to the adult rat hippocampus cannot be detected by proton magnetic resonance spectroscopy. Neural Regen Res. 2014;9:1616-20.

9. Gutiérrez J, Baraibar AM, Albiñana E, Velasco P, Solís JM, Hernández-Guijo JM. Methylmercury reduces synaptic transmission and neuronal excitability in rat hippocampal slices. Pflugers Arch. 2018;470:1221-30.

10. Altman J. Postnatal development of the cerebellar cortex in the rat. IV. Spatial organization of bipolar cells, parallel fibers and glial palisades. Comp Neurol. 1975;163:427-47.

11. Soriano E, Del Río JA, Martínez A, Supèr H. Organization of the embryonic and early postnatal murine hippocampus. I. Immunocytochemical characterization of neuronal populations in the subplate and marginal zone. J Comp Neurol. 1994;342:571-95.

12. Oomen CA, Bekinschtein P, Kent BA, Saksida LM, Bussey TJ. Adult hippocampal neurogenesis and its role in cognition. Wiley Interdiscip Rev Cogn Sci. 2014;5:573-87.

13. Committee on the Use of Complementary, and Alternative Medicine by the American Public Board on Health Promotion Disease Prevention Institute of Medicine. Complementary and Alternative Medicine in the United States. Washington DC: The National Academies Press; 2005.

14. Braun LA, Tiralongo E, Wilkinson JM, Spitzer O, Bailey M, Poole S, Dooley M. Perceptions, use and attitudes of pharmacy customers on complementary medicines and pharmacy practice. BMC Complement Altern Med. 2010;10:38.

15. Park BY, Min BS, Oh SR, Kim JH, Kim TJ, Kim DH, Bae KH, Lee HK. Isolation and anticomplement activity of compounds from Dendropanax morbifera. J Ethnopharmacol. 2004:90:403-8.

16. Park SY, Karthivashan G, Ko HM, Cho DY, Kim J, Cho DJ, Ganesan P, SuKim I, Choi DK. Aqueous extract of Dendropanax morbiferus leaves effectively alleviated neuroinflammation and behavioral impediments in MPTP-induced Parkinson's mouse model. Oxidative Med Cell Longev. 2018;2018:3175214.

17. Lee KY, Jung HY, Yoo DY, Kim W, Kim JW, Kwon HJ, Kim DW, Yoon YS, Hwang IK, Choi JH. Dendropanax morbifera Léveille extract ameliorates Dgalactose-induced memory deficits by decreasing inflammatory responses in the hippocampus. Lab Anim Res. 2017;33:283-90.

18. Kim W, Kim DW, Yoo DY, Jung HY, Nam SM, Kim JW, Hong SM, Kim DW, Choi JH, Moon SM, Yoon YS, Hwang IK. Dendropanax morbifera Léveille extract facilitates cadmium excretion and prevents oxidative damage in the hippocampus by increasing antioxidant levels in cadmium-exposed rats. BMC Complement Altern Med. 2014;14:428.

19. Kim W, Kim DW, Yoo DY, Jung HY, Kim JW, Kim DW, Choi JH, Moon SM, Yoon YS, Hwang IK. Antioxidant effects of Dendropanax morbifera Léveille extract in the hippocampus of mercury-exposed rats. BMC Complement Altern Med. 2015:15:247.

20. Kim W, Yim HS, Yoo DY, Jung HY, Kim JW, Choi JH, Yoon YS, Kim DW, Hwang IK. Dendropanax morbifera Léveille extract ameliorates cadmiuminduced impairment in memory and hippocampal neurogenesis in rats. BMC Complement Altern Med. 2016;16:452.

21. Choo GS, Lim DP, Kim SM, Yoo ES, Kim SH, Kim CH, Woo JS, Kim HJ, Jung JY. Anti-inflammatory effects of Dendropanax morbifera in ipopolysaccharide-stimulated RAW264.7 macrophages and in an animal model of atopic dermatitis. Mol Med Rep. 2019;19:2087-96.

22. Seo JS, Yoo DY, Jung HY, Kim DW, Hwang IK, Lee JY, Moon SM. Effects of Dendropanax morbifera Léveille extracts on cadmium and mercury secretion as well as oxidative capacity: a randomized, double-blind, placebo-controlled trial. Biomed Rep. 2016;4:623-7.

23. Kilkenny C, Browne WJ, Cuthill IC, Emerson M, Altman DG. Improving bioscience research reporting: the ARRIVE guidelines for reporting animal research. PLoS Biol. 2010;8:e1000412.

24. Hyun TK, Ko YJ, Kim EH, Chung IM, Kim JS. Anti-inflammatory activity and phenolic composition of Dendropanx morbifera leaf extracts. Ind Crop Prod. 2015;74:263-70

25. Yoo DY, Woo YJ, Kim W, Nam SM, Lee BH, Yeun GH, Yoon YS, Won MH, Park JH, Hwang IK. Effects of a new synthetic butyrylcholinesterase inhibitor, HBU-39, on cell proliferation and neuroblast differentiation in the hippocampal dentate gyrus in a scopolamine-induced amnesia animal model. Neurochem Int. 2011:59:722-8.

26. Yoo DY, Choi JH, Kim W, Nam SM, Jung HY, Kim JH, Won MH, Yoon YS, Hwang IK. Effects of luteolin on spatial memory, cell proliferation, and neuroblast differentiation in the hippocampal dentate gyrus in a scopolamine-induced amnesia model. Neurol Res. 2013;35:813-20.

27. Nam SM, Kim JW, Yoo DY, Jung HY, Chung JY, Kim DW, Hwang IK, Yoon YS. Hypothyroidism increases cyclooxygenase-2 levels and proinflammatory response and decreases cell proliferation and neuroblast differentiation in the hippocampus. Mol Med Rep. 2018;17:5782-8.

28. Paxinos $G$, Watson C. The rat brain in stereotaxic coordinates. Amsterdam: Elsevier Academic Press; 2007.

29. Nagy A, Delgado-Escueta AV. Rapid preparation of synaptosomes from mammalian brain using nontoxic isoosmotic gradient material (Percoll). J Neurochem. 1984:43:1114-23.

30. Rocha JB, Emanuelli T, Pereira ME. Effects of early undernutrition on kinetic parameters of brain acetylcholinesterase from adult rats. Acta Neurobiol Exp (Wars). 1993;53:431-7.

31. Grandjean P, Weihe P, White RF, Debes F, Araki S, Yokoyama K, Murata K, Sørensen N, Dahl R, Jørgensen PJ. Cognitive deficit in 7-year-old children with prenatal exposure to methylmercury. Neurotoxicol Teratol. 1997;19:417-28.

32. Graeme KA, Pollack CV Jr. Heavy metal toxicity, part I: arsenic and mercury. J Emerg Med. 1998;16:45-56.

33. Lapham LW, Cernichiari E, Cox C, Myers GJ, Baggs RB, Brewer R, Shamlaye CF, Davidson PW, Clarkson TW. An analysis of autopsy brain tissue from infants prenatally exposed to methymercury. Neurotoxicology. 1995;16:689-704.

34. Onishchenko N, Tamm C, Vahter M, Hokfelt T, Johnson JA, Johnson DA, Ceccatelli S. Developmental exposure to methylmercury alters learning and induces depression-like behavior in male mice. Toxicol Sci. 2007;97: 428-37.

35. Kim JM, Park SK, Guo TJ, Kang JY, Ha JS, Lee du S, Lee U, Heo HJ. Antiamnesic effect of Dendropanax morbifera via JNK signaling pathway on cognitive dysfunction in high-fat diet-induced diabetic mice. Behav Brain Res. 2016;312:39-54.

36. Aizawa K, Ageyama N, Yokoyama C, Hisatsune T. Age-dependent alteration in hippocampal neurogenesis correlates with learning performance of macaque monkeys. Exp Anim. 2009:58:403-7.

37. Rola R, Raber J, Rizk A, Otsuka S, VandenBerg SR, Morhardt DR, Fike JR. Radiation-induced impairment of hippocampal neurogenesis is associated with cognitive deficits in young mice. Exp Neurol. 2004;188:316-30.

38. Snyder JS, Hong NS, McDonald RJ, Wojtowicz JM. A role for adult neurogenesis in spatial long-term memory. Neuroscience. 2005;130:843-52.

39. Sokolowski K, Obiorah M, Robinson K, McCandlish E, Buckley B, DiCiccoBloom E. Neural stem cell apoptosis after low-methylmercury exposures in postnatal hippocampus produce persistent cell loss and adolescent memory deficits. Dev Neurobiol. 2013;73:936-49. 
40. Moretto MB, Lermen CL, Morsch VM, Bohrer D, Ineu RP, da Silva AC, Balz D, Schetinger MR. Effect of subchronic treatment with mercury chloride on NTPDase, $5^{\prime}$-nucleotidase and acetylcholinesterase from cerebral cortex of rats. J Trace Elem Med Biol. 2004;17:255-60.

41. Taupin P. Adult neurogenesis and neural stem cells as a model for the discovery and development of novel drugs. Expert Opin Drug Discov. 2010;5:921-5.

42. Kita Y, Ago Y, Higashino K, Asada K, Takano E, Takuma K, Matsuda T. Galantamine promotes adult hippocampal neurogenesis via $\mathrm{M}_{1}$ muscarinic and a7 nicotinic receptors in mice. Int J Neuropsychopharmacol. 2014;17:1957-68.

43. Kwon KJ, Kim MK, Lee EJ, Kim JN, Choi BR, Kim SY, Cho KS, Han JS, Kim HY, Shin CY, Han SH. Effects of donepezil, an acetylcholinesterase inhibitor, on neurogenesis in a rat model of vascular dementia. J Neurol Sci. 2014;347:66-77.

44. Girard C, Charette T, Leclerc M, Shapiro BJ, Amyot M. Cooking and co-ingested polyphenols reduce in vitro methylmercury bioaccessibility from fish and may alter exposure in humans. Sci Total Environ. 2018;616-617:863-74.

45. Wang X, Fan X, Yuan S, Jiao W, Liu B, Cao J, Jiang W. Chlorogenic acid protects against aluminium-induced cytotoxicity through chelation and antioxidant actions in primary hippocampal neuronal cells. Food Funct. 2017:8:2924-34.

46. Kwon SH, Lee HK, Kim JA, Hong SI, Kim HC, Jo TH, Park YI, Lee CK, Kim YB, Lee SY, Jang CG. Neuroprotective effects of chlorogenic acid on scopolamine-induced amnesia via anti-acetylcholinesterase and antioxidative activities in mice. Eur J Pharmacol. 2010;649:210-7.

47. Choi HJ, Park DH, Song SH, Yoon IS, Cho SS. Development and validation of a HPLC-UV method for extraction optimization and biological evaluation of hot-water and ethanolic extracts of Dendropanax morbifera leaves. Molecules. 2018;23:E650.

48. Franco JL, Posser T, Missau F, Pizzolatti MG, Dos Santos AR, Souza DO, Aschner M, Rocha JB, Dafre AL, Farina M. Structure-activity relationship of flavonoids derived from medicinal plants in preventing methylmercury-induced mitochondrial dysfunction. Environ Toxicol Pharmacol. 2010;30:272-8.

Ready to submit your research? Choose BMC and benefit from:

- fast, convenient online submission

- thorough peer review by experienced researchers in your field

- rapid publication on acceptance

- support for research data, including large and complex data types

- gold Open Access which fosters wider collaboration and increased citations

- maximum visibility for your research: over $100 \mathrm{M}$ website views per year

At $\mathrm{BMC}$, research is always in progress.

Learn more biomedcentral.com/submissions 